\title{
Hole-pair hopping in arrangements of hole-rich/hole-poor domains in a quantum antiferromagnet
}

\author{
Efstratios Manousakis \\ Department of Physics and MARTECH, Florida State University, Tallahassee, Florida, 32306, USA and \\ Department of Physics, Solid State Physics Section, University of Athens, Athens, Greece
}

(Dated: November 23, 2018)

\begin{abstract}
We study the motion of holes in a doped quantum antiferromagnet in the presence of arrangements of hole-rich and hole-poor domains such as the stripe-phase in high- $T_{C}$ cuprates. When these structures form, it becomes energetically favorable for single holes, pairs of holes or small bound-hole clusters to hop from one hole-rich domain to another due to quantum fluctuations. However, we find that at temperature of approximately $100 \mathrm{~K}$, the probability for bound hole-pair exchange between neighboring hole-rich regions in the stripe phase, is one or two orders of magnitude larger than single-hole or multi-hole droplet exchange. As a result holes in a given hole-rich domain penetrate further into the antiferromagnetically aligned domains when they do it in pairs. At temperature $T \sim 100 K$ and below bound pairs of holes hop from one hole-rich domain to another with high probability. Therefore our main finding is that the presence of the antiferromagnetic hole-poor domains act as a filter which selects, from the hole-rich domains (where holes form a self-bound liquid), hole pairs which can be exchanged throughout the system. This fluid of bound hole pairs can undergo a superfluid phase ordering at the above mentioned temperature scale.
\end{abstract}

PACS numbers: 74.20.-z,71.10.-w,71.45.Lr

\section{INTRODUCTION}

When an isotropic quantum antiferromagnet on an infinite square lattice, as described by the $t-J$ model without the inclusion of the long-range part of the Coulomb interaction, is doped with two holes a bound state is formed[1]. This bound state is not a sign of a pairing instability but that of a phase separation instability [2, 3] or possibly stripe ordering 4 . While in the high- $T_{c}$ cuprates stripes have been experimentally observed, at the theoretical level, there are two different scenarios to explain their formation. In the first scenario, when the antiferromagnet is doped with more than two holes they form a larger droplet and there is evidence [5, 6] that even for relatively small values of $J / t$ there is a critical electron density $n_{p s}(\mathrm{~J} / t)$ above which the system is separated into two phases: one phase in which the holes are bound into a hole-rich region and an all electron phase characterized by antiferromagnetic order. When one adds the longrange part of the Coulomb interaction this phase is forbidden because of the fact that the energy density of a droplet with finite charge density blows up as the droplet size goes to infinity. The system can accommodate this tendency for phase separation by forming a state which is made out of alternating microscopic regions of the two phases but with a zero net charge when averaged over a larger region. Such possible states are shown in Fig. 1 and in Fig. 2. In the second scenario the stripe-ordered state is obtained right away as the ground state of the $t-J$ model without the inclusion of the long-range Coulomb

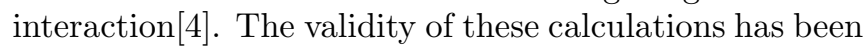
discussed $[8]$ and the issue has not been resolved unequivocally yet.

In this paper we consider the electronic system in a state characterized by alternating regions or domains with different charge such as the static or dynamic stripe phase (Fig. 1) or that of Fig. 2 or other structures which take care of the tendency for phase separation and the effects of long-range Coulomb repulsion; when such states form, it becomes energetically favorable for single holes or small bound-hole clusters to hop from one hole-rich domain to another due to quantum fluctuations. We show that the probability of bound hole-pair hop is one to two orders of magnitude larger than that of single-hole or multi-hole droplet hop between neighboring hole-rich regions. Therefore, pairs of holes in a given hole-rich domain prefer to penetrate further into the antiferromagnetically ordered domains relative to single or multi-hole clusters and they have a much higher chance to tunnel from one hole-rich domain to another. In particular, we find that below $T \sim 100 \mathrm{~K}$ these bound hole-pairs tunnel through the antiferromagnetic domains at high rates. Therefore, the system can be pictured as consisted of two inter-penetrating subsystems a) a subsystem of hole-rich domains in which the holes exist as a self-bound liquid and b) a fluid of bound hole pairs which exists in the antiferromagnetically ordered hole-poor domains. The latter fluid is composed of bound hole-pairs with a large characteristic binding energy scale of the order of the phase separation energy or the stripe formation energy scale. The main point of this paper is that the existence of the antiferromagnetic domains act as a filter which allows only pairs to exist over the entire system.

The second part of this paper includes a discussion which involves some speculation. The case of strong pairing correlations within each hole-rich strip and the role of the inter-strip coupling has been examined by other authors [6]. We wish to consider the limit where pairing within each hole-rich domain is weak at temperature of the order of $100 \mathrm{~K}$. We argue that the fluid of bound 
hole-pairs, which is selected by the presence of the intervening antiferromagnetic regions and exists throughout the system, can undergo a superfluid phase ordering at a relatively high critical temperature. In the limit of weak pairing within the hole-rich domains, this critical temperature associated with superfluid phase ordering depends on the pair effective mass inside the antiferromagnetic domain and the distance between the hole-rich domains.

\section{SINGLE-HOLE, HOLE-PAIR AND MULTI-HOLE TUNNELING}

In order to understand single-hole, hole-pair or multihole tunneling from one hole-rich domain (which could be a hole-rich strip or droplet) we will use a continuum model of holes of effective mass $m^{*}$, interacting through a mean field $V_{\text {eff }}$ introduced by the environment and the stripe-ordered or the dynamic-stripe configuration and via a residual hole-hole interaction $V$. The partition function for the system of $N$ holes can be written, using Feynman [9, 10] path integral formulation, as follows:

$$
\begin{aligned}
Z & =\frac{1}{N !} \sum_{P}(-1)^{[P]} \int_{\vec{x}_{i}(\beta)=P \vec{x}_{i}(0)} \prod_{i=1}^{N} \mathcal{D} x_{i}(\tau) e^{-S_{e f f}} \\
S_{e f f} & =\int_{0}^{\beta} d \tau\left[\sum_{i=1}^{N}\left(\frac{m^{*}}{2 \hbar^{2}}\left(\frac{d \vec{x}_{i}(\tau)}{d \tau}\right)^{2}+V_{e f f}\left(\vec{x}_{i}(\tau)\right)\right)\right. \\
& \left.+\sum_{i<j} V\left(\vec{x}_{i}(\tau)-\vec{x}_{j}(\tau)\right)\right]
\end{aligned}
$$

where the sum is over all $N$ ! hole-permutations $P$ and $[P]$ is the order of permutation. The integration is over paths $\vec{x}_{i}(\tau)$ in imaginary time $\tau$. The final configuration $\vec{x}_{i}(\beta)$ at $\tau=\beta$ is any permutation $P \vec{x}_{i}(0)$ of the initial hole-positions.

Let us consider a state of alternating hole-rich and antiferromagnetically aligned hole-poor domains. When these alternating regions order they form the stripeordered state shown in Fig. 1 (a). In addition we will keep in mind other inhomogeneous configurations such as the one in Fig. 2. In the case of the stripe state, each hole experiences a potential $V_{\text {eff }}$ with a profile shown in Fig. 1(b). We wish to understand the tunneling of one or more holes from one hole-rich domain to the nearest hole-rich domain. First, let us consider the motion of a single-hole and estimate the factor $\exp \left(-S_{\text {eff }}\right)$ for a hole displacement from the boundary of a hole-rich domain by a distance $x$ within imaginary time $\tau$ inside the antiferromagnetic hole-poor domain. At the beginning the hole is near the boundary of the hole-rich with the hole-poor region and so an estimate of the $S_{\text {eff }}$ is given as follows

$$
S_{e f f}^{(1)}(x, \tau)=\frac{m_{1}^{*}}{2 \hbar^{2}} \frac{x^{2}}{\tau}+V_{1} \tau .
$$

Here $m_{1}^{*}$ is the hole effective mass inside the antiferromagnet (hole-poor region), $V_{1}$ is the difference between the hole energy when the hole is in the hole-poor region and the hole energy when the hole is in the hole-rich region. This is determined by the presence of an effective barrier imposed by the existence of the two different domains and more significantly by the hole-hole interaction. A single hole sticks to the hole-rich region strongly while a pair of holes sticks to the rest of the hole-rich region relatively weakly. This is shown on the basis of the $t-J$ model later below. This means that the value of the barrier $V_{1}$ for a single hole to enter the antiferromagnetic region is significantly larger than the barrier $V_{2}$ which is experienced by a pair of holes. The path with the most significant contribution to the partition function is that which corresponds to a characteristic time scale $\tau$ (the time for which the hole stays in the hole-poor region) such that $S^{(1)}(x, \tau)$ for a given $x$ is minimum. The minimum of the action (2) is obtained for

$$
\tau_{0}=x \sqrt{\frac{m_{1}^{*}}{2 \hbar^{2} V_{1}}}
$$

and for this value of $\tau$ the action is given by

$$
S_{\min }^{(1)}=\sqrt{\frac{2 m_{1}^{*} V_{1}}{\hbar^{2}}} x
$$

This gives the well-known result for the quantum mechanical penetration probability $\left(e^{-S^{(1)}}\right)$. Therefore the "penetration" depth inside the hole-poor region (the value of $x$ where $\left.e^{-S^{(1)}}=1 / e\right)$ is given by

$$
\lambda_{1}=\frac{\hbar}{\sqrt{2 m_{1}^{*} V_{1}}} .
$$

As we will show below a pair of holes sticks much less strongly to the hole-rich region; thus, the difference in energy $V_{2}$ for the pair in the hole-rich and hole-poor domains is much smaller than $V_{1}$. The penetration depth for hole-pair or multi-hole tunneling is given by

$$
\lambda_{N}=\frac{\hbar}{\sqrt{2 m_{N}^{*} V_{N}}}
$$

We would like to estimate these penetration depths and probabilities on the basis of results obtained by Green's function Monte Carlo simulation of the $t-J$ model 1 , 5 , 11, 12.

We consider the $t-J$ model in the parameter range where phase separation was found [3, 5, 12. Starting from the undoped insulator we introduce $N$ holes in a system of $N_{s}=L \times L$ sites. The ground state of the system keeping $N$ finite and $L \rightarrow \infty$ is expected to be a two-dimensional droplet with energy per hole given by $e(N)=(E(N)-E(0)) / N$. The situation is shown in Fig. 3 for $J / t=1$ and in Fig. 4 for $J / t=0.4$. The circles with the error bars give the energy per hole $e(N)$ as a function of the number of holes. The solid line in both figures denotes the minimum of the energy per hole as 


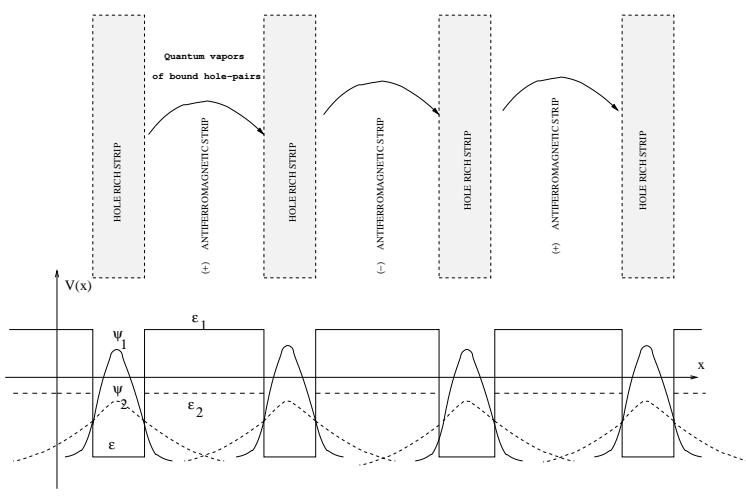

FIG. 1: (a) A striped ordered state, i.e., alternating strips of the hole-rich and the hole-poor phases. Two successive holepoor domains are separated by a pi-phase shift. In general we consider the case of dynamic stripes where while these alternating domains exist there is no stripe ordering. (b) The effective potentials experienced by a single hole (solid line) and bound hole-pair when attempting to tunnel from one hole-rich region to another. The corresponding wave functions of the single-hole and bound hole-pair are also shown schematically as $\psi_{1}$ and $\psi_{2}$.

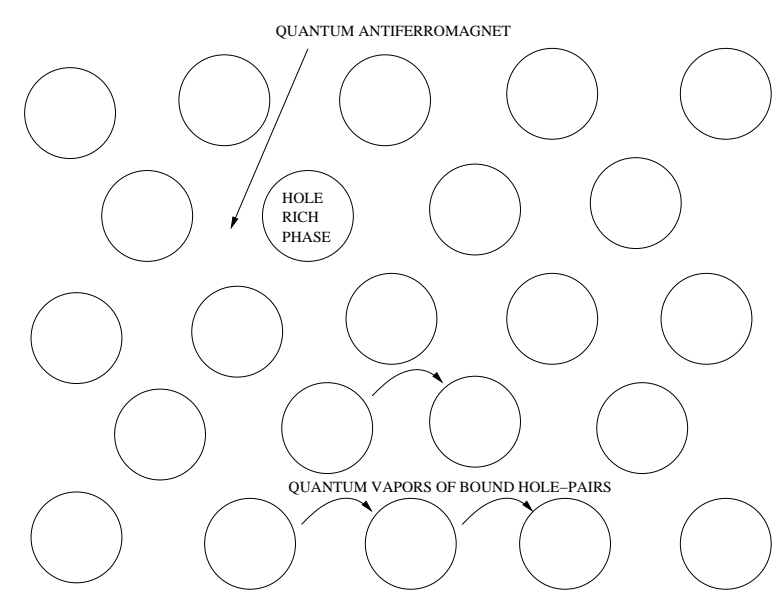

FIG. 2: A hexagonal superlattice formed from droplets of the hole-rich phase inside an antiferromagnetic background.

a function of the hole density, namely the energy of the phase separated state $e_{p s}$. The energy per hole $e(N)$ is always higher than $e_{p s}$. The quantity $V_{N}$ discussed previously, is given by $\left(e(N)-e_{p s}\right) N$. The important facts to notice is that a) the energy per hole for the bound-pair of holes is quite close to that of the energy per hole in the phase separated state and b) introducing two holes inside the quantum antiferromagnet lowers the energy per hole significantly relative to the single hole case and further introduction of holes leads to formation of droplets without as significant gain in binding energy. This implies that it is far easier to separate a hole-pair than a singlehole from the hole-rich region. The energy of a hole-rich droplet inside a quantum antiferromagnet has an additional contribution due to long-range Coulomb repulsion. It is clear than when we switch on this interaction, the

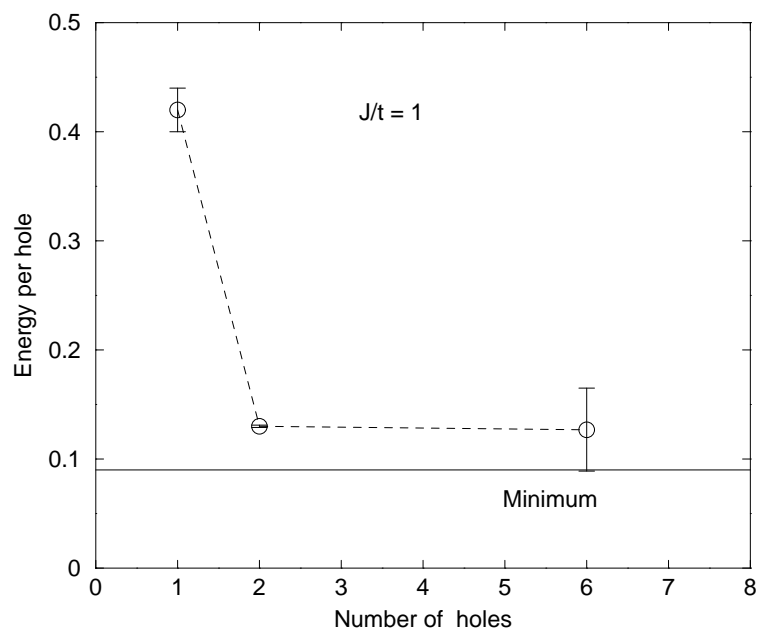

FIG. 3: The energy per hole of a hole-rich droplet in an antiferromagnetic background as a function of the number of holes at $J / t=1$ (dashed line and solid circles). The solid line is the value of the minimum value of the energy per hole as a function of the hole density for $J / t=1$

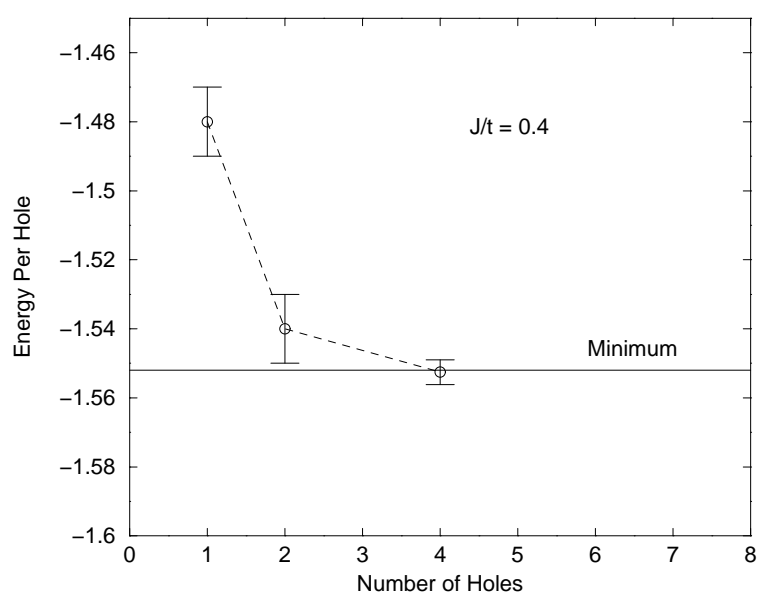

FIG. 4: The energy per hole of a hole-rich droplet in an antiferromagnetic background as a function of the number of holes at $J / t=0.4$ (dashed line and solid circles). The solid line is the value of the minimum value of the energy per hole as a function of the hole density for $J / t=0.4$

macroscopically phase separated state cannot be realized because the energy per hole has positive infinite energy. However, a state of microscopic phase separation is allowed because there is no net charge on a large scale and examples of such states are given in Fig. 1 and Fig. 2 . The effect of this long-range interaction does not alter our conclusion and it will be discussed later.

While $m_{N}^{*}$ increases almost linearly with the number of holes $N$ 13], the function $V_{N}=\left(e(N)-e_{p s}\right) N$ drops sharply at the value $N=2$. As a result the combination of $m_{N}^{*} V_{N}$ which occurs in Eq. 66 causes a peak in $\lambda_{N}$ at $N=2$. The fact that the largest $\lambda_{N}$ corresponds to bound hole-pairs implies that when stripes form, because 
of the overlap of the wave-function of hole clusters within each hole-rich region with the wave-function of a neighboring hole-rich strip, the number of bound hole-pairs which will be shared among all strips is much larger than the number of single holes or the number of any other multi-hole clusters shared by all the strips. In addition using values for $V_{N}$ and $m_{N}^{*} / m$ obtained from numerical studies of the $t-J$ model [1, 5, 11, 12] and the Hubbard model 13 we find that $\lambda_{2}$ is of the order of $10 \AA$ which is of the inter-strip distance when the stripes order. In the next section we show that the contribution of such tunneling configurations (in which hole-pairs cross from a hole-rich strip to a neighboring hole-rich strip through the intervening antiferromagnetic hole-poor domains) to the path integral (11) become very significant at and below a temperature of approximately $100 \mathrm{~K}$.

Tunneling of hole-pairs between neighboring strips contribute to the path-integral (11) by a factor $\exp \left(-S_{\text {pair }}(d, \beta)\right)$ while when a single-hole crosses from one hole-rich strip to a neighboring one through the antiferromagnetic domain it contributes to the path integral by an amount $\exp \left(-S_{1}(d, \beta)\right)$. The ratio of these contributions is given by $\exp \left(-\Delta S_{12}(d, \beta)\right)$ where $\Delta S_{12}(d, \beta)$ can be estimated as

$$
\Delta S_{12}(d, \beta)=\frac{m_{1}^{*}-m_{2}^{*}}{2 \hbar^{2}} \frac{d^{2}}{\beta}+\left(V_{1}-V_{2}\right) \beta .
$$

Taking $T=100 K$ and using the available results for $J / t=0.4$, namely, $V_{1} \sim 0.07 t, V_{2} \sim 0.02 t$, and the values of $m_{1}^{*} / m_{0} \sim 6.5, m_{2}^{*} / m_{0} \sim 22.5$ (as obtained by Trugman 13 for $U / t=10$ ) and the value $d \sim 16 \stackrel{\circ}{\text { (a }}$ typical distance between strips in a stripe-ordered state) we find that $S_{1}-S_{\text {pair }} \sim 4$. This implies that the contribution of a single hole $e^{-S_{1}}$ is approximately 1-2 orders of magnitude smaller than that of a bound hole-pair at the above mentioned temperature.

In order to give an accurate determination of such transition rates for hole-pairs we need a) a more accurate determination of the pair binding energy difference relative to the energy of the phase separated state and b) to know the effect of the long-range Coulomb repulsion on the binding energy of two holes inside an antiferromagnetic domain relative to the energy of these two holes when they are in the hole-rich domain. The value we used above, namely $V_{2} \sim 0.02 t$ will be significantly reduced in the presence of the long-range (LR) Coulomb interaction. The turning on of the LR Coulomb interaction will reduce the binding energy of a bound hole-pair to the rest of the hole-rich region. The stripes result from the competition between the tendency of the system for phase separation and the LR Coulomb interaction. Therefore the hole-rich region contains just enough holes to keep the balance between these two opposing tendencies. This implies that the binding energy of a bound pair to the rest of the hole-rich domain relative to the case where the hole-pair is inside the antiferromagnetic hole-poor region is very small. This is in agreement with the findings of Arrigoni et al.14. who studied the $t-J$ model and they added the
LR Coulomb interaction using a combination of density matrix renormalization group method for the short-range part and a Hartree approximation to take into account the LR part. When they added the LR Coulomb interaction they find enhanced superconducting correlations which was associated to the enhancement of pair tunneling between stripes.

\section{SUPERFLUIDITY OF THE GAS OF BOUND HOLE-PAIRS}

This section includes a discussion which is speculative. If pairing correlations within a given hole-rich stripe domain are strong we are in a limit which has been considered by other authors [6]. In such case a weak inter-strip coupling can give rise to a high $T_{C}$ and superconductivity competes with charge density wave ordering. Here we wish to examine the case where pairing correlations within a given strip are weak at temperature of the order of $100 K$. Strong pairing correlations exist in ladders but when stripes occur in a two-dimensional system it is uncertain that such one-dimensional pairing correlations are strong at this temperature.

If we neglect $V_{2}, S_{\text {pair }} \sim\left(m_{2}^{*} d^{2} / 2 \hbar^{2}\right) K_{B} T$ and it is of order of unity at $T \sim 100 K$. At around this temperature the system of such hole-pairs acquires long-range phase coherence and non-zero winding number due to long chains of exchanges of hole-pairs between neighboring strips. The most significant contributions to the partition function (11) at temperature of the order of $100 \mathrm{~K}$ and below can be written as

$$
\begin{aligned}
Z= & \frac{1}{N_{p} !}\left(\frac{m_{2}^{*}}{2 \pi \hbar^{2} \beta}\right)^{\frac{3 N_{p}}{2}} \int \prod_{i=1}^{N_{p}} d \vec{z}_{i} g\left(\vec{z}_{1}, \vec{z}_{2}, \ldots, \vec{z}_{N_{p}}\right) \\
& \sum_{P} \exp \left(-\frac{m_{2}^{*}}{2 \beta \hbar^{2}} \sum_{i}^{N_{p}}\left(\vec{z}_{i}-P \vec{z}_{i}\right)^{2}\right) .
\end{aligned}
$$

where $N_{p}=N / 2$ is the number of hole pairs in the system with a distribution governed by the function $g\left(\vec{z}_{1}, \vec{z}_{2}, \ldots, \vec{z}_{N_{p}}\right)$ giving the probability for a given configuration $\left\{\vec{z}_{i}\right\}$ of the bound hole pairs as in a Bose fluid. There is a sum over all possible permutations of hole-pairs. Here we have assumed that the path integration over all trajectories in Euclidean-time in the path-integral of Eq. (11) has been carried out 10. Let us consider a typical displacement of a hole inside the hole-rich domains which is associated with the factor $f_{1}=\exp \left(-m_{1}^{\prime}|\vec{x}|^{2} / 2 \hbar^{2} / \tau-U_{1}(\tau)\right)$ where $U_{1}(\tau)$ is the interaction part of the action and $m_{1}^{\prime}$ the hole effective mass inside the hole-rich domains. Similarly when a pair of holes moves a similar typical distance between pairs inside the hole-rich domain we can associate a factor $f_{2}=\exp \left(-m_{2}^{\prime}|\vec{x}|^{2} / 2 \hbar^{2} / \tau-U_{2}(\tau)\right)$. For hole displacements of the order of the inter-hole distance in the hole-rich domains, both $f_{1}$ and $f_{2}$ become of order of unity for significantly smaller values of the imaginary 
time $\tau$ (higher temperatures) as compared to the time scale required for hole-pair exchange between neighboring hole-rich domains. Namely, in order to provide an optimum hole distribution within the strips the holes, in these regions, adjust their positions in shorter times scales; the effects discussed previously which involve tunneling of pairs of holes between two neighboring holerich regions are longer-time effects. Therefore we have assumed that at such lower temperature where these latter effects become energetically favorable, we can integrate out these short times scale configurations; the holes within each strip have enough time to relax to a distribution $g\left(\vec{z}_{1}, \ldots, \vec{z}_{N_{p}}\right)$ which is experienced by the bound hole-pairs as they move from one hole-rich domain to another. This distribution $g$ describes the stripe ordering and the charge-stripe correlations. We have eliminated the single-hole degrees of freedom even within a strip. We consider the hole-rich domains as made of pairs of holes with the same charge distribution because what is important in describing our ideas is the movement of the gas of bound hole pairs in the space between hole-rich domains. The space occupied by the hole-rich strips plays only the role of a reservoir from where these bound hole pairs emerge. The characteristic time-scale associated with the motion of these degrees of freedom in imaginary time is much shorter than the inverse temperature at which tunneling of bound hole-pairs between hole-rich domains becomes likely.

When a bound hole-pair is exchanged between neighboring stripes, it leads to a factor $f=$ $\exp \left(-m_{2}^{*} /\left(2 \beta \hbar^{2}\right)\left(\vec{z}_{i}-\vec{z}_{j}\right)^{2}\right)$. This can occur anywhere in the system between any two neighboring stripes. These pair-hole movements cannot occur in an uncorrelated manner because when the pairs move to another holerich domain they disturb the balance of charge. Such unbalanced configurations correspond to large values of the effective action and they will not contribute to the observables. When closed rings of exchanges occur, namely cyclic permutations of bound-pairs, any transient charge imbalance should be eliminated. Below a certain temperature, of the order of $100 \mathrm{~K}$ as shown above, the factor $f$ becomes of the order of unity and then such coherent multiple exchanges can occur which lead to the off-diagonallong-range order. The arguments which lead to this last part of the picture are very similar to those discussed by Feynman 10 for the case of liquid helium- 4 .

Since the partition-function (8) describes a system of purely boson degrees of freedom, we can gain additional support for this picture by discussing what happens in a simpler model. We consider a system of bosons interacting via a Van der Waals interaction and we add a long-range $\alpha / r$ Coulomb-like interaction with a very small value for $\alpha$. First let us consider the case of $\alpha=0$, namely that of a pure self-bound boson liquid on a two-dimensional substrate. Such a system has a physical realization, namely, it describes liquid helium-4 on a substrate such as graphite. The pure liquid neutral helium-4 on a 2D smooth substrate (without sub- strate corrugations) with periodic boundary conditions has been studied 16 using path-integral Monte Carlo simulation 15 . It was found that for values of the $2 \mathrm{D}$ density below the $2 \mathrm{D}$ equilibrium density and at low temperature the system forms a 2D liquid droplet with non-zero winding number (superfluid density) for densities well inside the phase separated region. Let us discuss what we expect to find if we introduce the long-range $\alpha / r$ interaction term with a small value of $\alpha$ so that a selfbound droplet with at least two atoms exists as a result of the competition of the long-range and the Van der Waals attraction. We expect that the uniformity of the system will be spoiled by the presence of this long-range interaction and droplets of certain size will be formed. The system optimizes the number of atoms $N_{0}$ in a droplet, namely, the binding energy per atom will be very small compared to the magnitude of the van der Waals interaction. This implies that an atom in any charged droplet will be only very weakly bound to the droplet and this will allow a non-zero winding number caused from exchanges of atoms between these droplets. An estimate of the temperature scale where such exchanges occur is

$$
K_{B} T_{C} \sim \frac{\hbar^{2}}{2 m d^{2}}
$$

where $d$ is the average distance between neighboring droplets. We expect to find a similar result if one introduces a weak external potential so that the chargedsuperfluid is confined in a charge-stripe-like configuration; namely, the off-diagonal-long-range order sets in at a temperature of the above mentioned magnitude where $d$ is the distance between successive hole-rich domains.

\section{CONCLUDING REMARKS}

We have considered the state in which alternating holerich and hole-poor (antiferromagnetically ordered) domains form in a strongly correlated electron system.

Here we have used existing numerical results on the $t-J$ model to show that the presence of antiferromagnetically ordered regions between such hole-rich domains acts as filter which allows only pairs of holes to jump from one hole-rich domain to a nearest neighboring one at high rates. This implies that effectively there is a fluid of bound hole pairs which can move through the entire system.

We further discussed a speculative point of view. We considered the limit in which pairing correlations within each hole-rich domain are weak which implies that such a domain is characterized by a small superconductivity ordering temperature scale. In this case we argued that because of the existence of the previously discussed fluid of bound hole-pairs, the characteristic energy scale associated with phase coherence between such pairs is determined by the distance between neighboring hole-rich domains and the pair effective mass inside the antiferromagnetically ordered domains. 


\section{ACKNOWLEDGEMENTS}

We would like to thank B. I. Halperin, C.S. Hellberg and S. A. Kivelson for useful discussions and comments on the original draft of this paper.
[1] M. Boninsegni and E. Manousakis, Phys. Rev. B 47, 11897-11904 (1993).

[2] V. J. Emery, S. A. Kivelson, and H.Q. Lin, Phys. Rev. Lett. 64, 475 (1990).

[3] C. S. Hellberg and E. Manousakis, Phys. Rev. Lett. 78, 4609 (1997).

[4] S. R. White and D. J. Scalapino, Phys. Rev. B 55, 6504 (1997); 60, R753 (1999); Phys. Rev. Lett. 81, 3227 (1998).

[5] C. S. Hellberg and E. Manousakis, Phys. Rev. B 61, 11787 (2000). See also references and comparison of various numerical results therein.

[6] E. W. Carlson, V. J. Emery, S. A. Kiveslon, D. Ograd, "The Physics of Conventional and Unconventional Superconductors" ed. by K. H. Bennemann and J. B. Ketterson (Springer-Verlag).

[7] S. A. Kivelson, E. Fradkin and V. J. Emery, Nature (Lon- don) 393, 550 (1998).

[8] C. S. Hellberg and E. Manousakis, Phys. Rev. Lett. 83, 132 (1999); S. R. White and D. J. Scalapino, Phys. Rev. Lett. 84, 3021 (2000).

[9] R. P. Feynman, Phys. Rev. 90, 1116 (1953).

[10] R. P. Feynman, Phys. Rev. 91, 1291 (1953).

[11] M. Boninsegni and E. Manousakis Phys. Rev. B 46, 560563 (1992).

[12] F. B. Calandra, F. Becca and S. Sorella, Phys. Rev. Lett. 81, 5185 (1998).

[13] S. Trugman, Phys. Rev. B 37, 1597 (1988).

[14] E. A. Arrigoni, A. P. Harlu, W. Hanke, B. Brendel and S. A. Kivelson, Phys. Rev. B 65, 134503 (2002).

[15] D. M. Ceperley, Rev. Mod. Phys. 67, 279 (1995).

[16] M. Pierce and E. Manousakis, Phys. Rev. B 59, 3802 (1999). 